\title{
Amanda Labarca (1886-1975) y sus referencias psicológicas en el contexto educativo en Chile*
}

\author{
Amanda Labarca (1886-1975) and her Psychological \\ References in the Educational Context in Chile
}

Recibido: diciembre 12 de 2013 | Revisado: junio 24 de 2014 | Aceptado: julio 28 de 2014

\author{
GonZALO SALAS ** \\ Universidad Católica del Maule, Chile \\ RODOLFO MARDONES **** \\ Universidad Santo Tomás, Chile \\ Miguel GALLEGOS**** \\ Universidad Nacional de Rosario, Argentina
}

FERNANDO P. PONCE ******

Universidad de Talca, Chile

Para citar este artículo: Salas, G., Mardones, R., Gallegos, M., \& Ponce, F. P. (2014). Amanda Labarca (1886-1975) y sus referencias psicológicas en el contexto educativo en Chile. Universitas Psychologica, 13(5), 2059-2068. http://dx.doi.org/10.11144/ Javeriana.upsy13-5.alrp

Agradecimientos: El primer autor agradece el apoyo del Departamento de Psicología de la Facultad de Ciencias de la Salud, Universidad Católica del Maule.

** Contacto principal para correspondencia editorial. Académico de planta. Departamento de Psicología. Académico e investigador Departamento de Psicología, Universidad Católica del Maule. Representante Nacional (Chile) Sociedad Interamericana de Psicología (SIP)

Correo Electrónico: gsalas@ucm.cl

**** Académico e investigador, Escuela de Psicología, Universidad Santo Tomás, Los Ángeles.

Correo electrónico: rodolfomardonesba@santotomas.cl

***** Académico e investigador, Facultad de Psicología, Universidad Nacional de Rosario, CONICET. Correo electrónico: maypsi@yahoo.com.ar

******** Investigador Asociado, Laboratorio de Psicología Experimental, Facultad de Psicología, Universidad de Talca.

Correo electrónico: fponce@utalca.cl

\section{RESU MEN}

Amanda Labarca fue una connotada educadora chilena que vivió entre los años 1886 y 1975, cuya contribución e influencia a la psicología ha sido recientemente reconocida en Chile. El presente trabajo se propone dar a conocer sus antecedentes biográficos y académicos, por medio de una revisión documental, para luego contextualizar el entramado sociopolítico de la época. Finalmente, se describen sus principales referencias psicológicas en el ámbito educativo. En su transitar, viajó constantemente a Estados Unidos empapándose del trabajo de William James y John Dewey, dos de sus principales maestros, a quienes admiró y dio a conocer sus teorías.

Palabras clave

Amanda Labarca; historia de la psicología; educación y pedagogía

\section{A B S T R A C T}

Amanda Labarca was a renowned teacher that was born in 1886 and died in 1975. She was the first female university teacher in Chile. Her contribution and influence in psychology have been recently recognised. The objective of the present work is to show her personal and academic history by means of an evidence review, to provide a context for the socio-political period. Finally, her main psychological references, regarding the educational area, are described. Due to her frequent visits to USA, she was steeped in William James and John Dewey's work, her main masters, to whom she admired and tried to spread their theories.

Keywords

Amanda Labarca; history of psychology; education and pedagogy 
La historia de la psicología se ha caracterizado por un marcado sesgo androcéntrico, a la hora de reconocer y visualizar los avances significativos en esta disciplina, apartando de sus registros a aquellas mujeres que han realizado importantes contribuciones en materia de investigación y reflexión teórica. Esta omisión ha llevado a un progresivo olvido de varias psicólogas y ha sido responsable de un evidente desconocimiento de sus aportes (Bohan, 1993a, 1993b; Winkler, Magaña, \& Wolff, 2004). Tal situación ha favorecido la perpetuación del mito de una "historia de la psicología sin mujeres", que se ha venido sosteniendo a través de las generaciones, lo cual se contrapone a los aportes desarrollados por las mujeres durante la institucionalización de la psicología como ciencia, desde finales del siglo XIX (García, 2003, 2010).

La historiadora Gerda Lerner, planteó que la necesidad de resituar a las mujeres en la historia de la psicología no debería ser el resultado de un proceso compensatorio que apunte a recuperar nombres perdidos en el naufragio femenino de la travesía histórica de la disciplina, ni tampoco un recogimiento de sus contribuciones científicas en función de los parámetros y estándares dominantes en la psicología, sino más bien este reposicionamiento debiera regenerar y a su vez reconstruir una historia de las mujeres en psicología "en sus propios términos", recuperando contextualmente sus nombres y sus contribuciones infravaloradas, a través de la narración de sus experiencias y situaciones marcadas por una división sexista (García, 2005).

Favorablemente, en los últimos años ha crecido el interés por recuperar la figura de las mujeres en la historia de la psicología, y en esa dirección se vienen desarrollando aportes muy significativos (García, 2010; Jacó-Vilela, Santos, \& Barbosa, 2008; Jacó-Vilela et al., 2007; Moradi \& Townsend, 2006; Ostrovsky, 2010; Scott et al., 2012). En América Latina, se destaca el trabajo psicológico de las emigradas españolas Mercedes Rodrigo en Colombia (Ardila, 1973, 1988), Fernanda Monasterio (Carpintero, 2012; Quintana, \& Feldman, 2007) y Erminda Benítez de Lambruschini en Argentina, impulsora y creadora de la primera carrera de psicología en Argentina, en 1955 (Ascolani, 1988; Gallegos, 2005; Gallegos \& Berra, 2012).

En el caso de la historia de la psicología en Chile, los trabajos críticos desde la perspectiva de género evidenciaron una ausencia de referencias femeninas en la historia de la disciplina (Magaña, Wolff, \& Winkler, 1998; Miranda \& Navarro, 1995). Sin embargo, dicha situación fue posteriormente contrarrestada con una serie de investigaciones que han intentado reposicionar a diversas mujeres chilenas y sus aportes en el desarrollo de la psicología (Winkler, 2007, 2012; Winkler, Magaña, \& Wolff, 2001), así como también por varios estudios comparados entre destacadas psicólogas en Chile y Estados Unidos (Winkler, 2004; Winkler, Pasmanik, Wolff, \& Reyes, 2007; Wolff, Winkler, \& Alvear, 2010).

Los trabajos descritos anteriormente se han focalizado en el estudio y divulgación de las contribuciones de psicólogas nacionales y americanas, sin embargo, se observa una escasez de trabajos que muestren los aportes de mujeres "no psicólogas" a la disciplina, tanto por recibir influencias desde la psicología, como por la divulgación y aplicación de las ideas psicológicas en Chile. Uno de estos casos corresponde a la educadora chilena Amanda Labarca (1886-1975).

Hasta el momento, la literatura sobre Amanda Labarca ha reparado en su vida y obra (Reyes, Vargas, \& Meza, 2002), destacando su rol en el campo de la educación y en el activismo político de las mujeres (Guil \& Vera, 2010), así como en la historia de la enseñanza en Chile (Núñez, 2002). Sin embargo, no se conocen estudios que tiendan a recuperar su participación en la difusión y circulación del conocimiento psicológico en el país.

El presente trabajo se propone recuperar algunas de las referencias psicológicas con las cuales Amanda Labarca situó su trabajo a principios del siglo XX en Chile. Para avanzar con esta recuperación historiográfica, se realizó una investigación documental de fuentes primarias de la autora en los archivos de la Biblioteca Nacional de Chile y fuentes complementarias, tales como periódicos, libros, actas y artículos que en su conjunto, permitieron esbozar un esquema de la contribución de Amanda Labarca para la divulgación de la psicología en Chile. 


\section{Aspectos biográficos y académicos}

Amanda Labarca Hubertson nació en la ciudad de Santiago el 22 de Diciembre de 1886 y es considerada como una de las grandes educadoras de Chile e Hispanoamérica, durante la primera mitad del siglo XX. Uno de los aspectos más relevantes que se observa cuando se estudian los diversos aspectos de su vida y obra, fue su capacidad de insertarse en un mundo abiertamente hostil a las actividades femeninas.

Su trayectoria profesional fue nítida, alcanzó el grado de Bachiller a los quince años. Entre los años 1902 y 1904, continuó sus estudios en el recién creado Instituto Pedagógico de la Universidad de Chile, donde recibió la enseñanza de eminentes profesores germanos que llegaron al país desde el año 1889 (Salas, 2012; Salas \& Lizama, 2013). A los 18 años obtuvo con distinción su diploma de nobleza intelectual, el entonces nuevo título heroico de Profesor de Estado (Munizaga, 1991). En esa fecha, 1905, se recibieron trece profesores de los cuales solo tres fueron mujeres (Salas, 1996). Posteriormente, prosiguió sus estudios en la Universidad de Columbia en Estados Unidos (Palma, 1976) y en La Sobornne, en París.

Su labor en la educación se inició como directora del Liceo № 5 de Niñas de Santiago y, posteriormente, continuó en el entorno universitario (De la Fuente, 1979, 2000), en donde, tras someterse a rigurosas exigencias reglamentarias, se la designó en 1922 como Profesora Extraordinaria de Psicología en el Instituto Pedagógico de donde años atrás había egresado. Al año siguiente, ocupó el cargo de Profesora de Filosofía, sin perjuicio de los cursos de educación en los que sirvió posteriormente.

El ascenso de Amanda como primera mujer académica en Latinoamérica ha sido un hecho reconocido por varios historiadores (Barr-Melej, 2001; Salas, 1995; Vargas, 2006), educadores (Fernández, 2007; Munizaga, 1991; Pinto, 1990) y periodistas (Portugal, 1993). Asimismo, otros autores no solo han subrayado la inclusión de Amanda como la primera mujer que asumió un puesto catedrático en la Universidad de Chile, sino, además, la ubican como la primera mujer en asumir una cátedra uni- versitaria de psicología (Reyes et al., 2002; Winkler, 2007). En su extenso periplo académico, fue miembro del Consejo Universitario de la Universidad de Chile durante 20 años, ejecutando una labor que sobrepasó los límites nacionales, se extendió por América Latina y repercutió en el Secretariado de la Mujer en las Naciones Unidas, desde donde favoreció el progreso y la visibilidad de las mujeres, puesto que la vida de las vencedoras parecía "más trágica que épica”, donde sus triunfos estaban más emparentados con lágrimas y renuncias, que en un futuro cargado de esperanzas y realizaciones (Palma, 1976). Finalmente, a la edad de 88 años y tras largas e incansables jornadas de trabajo, falleció en Santiago el 2 de enero de 1975 (Muñoz, 1998), dejando una huella indeleble en diversos aspectos del desarrollo educativo y social de Chile.

En cuanto a sus obras, fue autora de diversos manuscritos, textos de enseñanza, estudios especializados e innumerables artículos de prensa sobre los más diversos tópicos, entre los que se destacan sus reflexiones sobre temas de carácter nacional o latinoamericano, plasmados en La enseñanza secundaria en los Estados Unidos (Labarca, 1919) y La evolución de la segunda enseñanza (Labarca, 1938), junto con un análisis crítico de la situación de la mujer en la sociedad, cuyos lineamientos se aprecian en la obra Feminismo contemporáneo (Labarca, 1947).

La educadora también dedicó parte de su obra a un análisis histórico de la realidad social y educacional del país, en trabajos como Mejoramiento de la vida campesina (Labarca, 1936), Historia de la enseñanza en Chile (Labarca, 1939), Bases para una politica educacional (Labarca, 1944) y Realidades y problemas de nuestra enseñanza (Labarca, 1953). En general, estas obras reflejan aspectos centrales del pensamiento de la educadora y son fundamentales para comprender el marco de referencia que guió su vida como educadora y política. No obstante, también existe en la obra de Amanda varias referencias ligadas a los estudios psicológicos durante su tiempo de actividad académica e intelectual, las cuales permiten complementar y profundizar una comprensión sobre su entendimiento de la educación, en trabajos como Nuevas orientaciones de la enseñanza (Labarca, 1927a) y Lecciones de filosofía (Labarca, 1927b). 


\section{Entramado sociopolítico y su preocupación por la mujer}

Amanda vivió entre dos siglos, desde las postrimerías del XIX hasta la década de los años setenta del siglo XX (Salas, 1996). En sus casi noventa años de existencia, se produjeron en el país y en el mundo significativos cambios sociales y políticos que ella observó inteligentemente, y en muchos casos, contribuyó a que se desarrollaran. Durante la primera mitad del siglo XIX, el Chile independiente se esforzó por construir las bases tanto materiales como intelectuales de la República. Fue un período marcado por el fenómeno del cambio y la continuidad, por la ligazón del orden con la libertad, tan propios de los países latinoamericanos en nacimiento (Dager, 2003).

En diciembre de 1886, en el Santiago de fines del siglo XIX, la ciudad tenía alrededor de 300.000 habitantes, se conservaban casas de adobe de una sola planta, angostos callejones aledaños y estrechas calles adoquinadas (Salas, 1996). Ya a comienzos del siglo XX, quedaba en evidencia que la modernización estaba presente, aunque rodeada de un mar de contradicciones fomentadas por las oligarquías imperantes, haciendo manifiestas las siguientes situaciones: modernización con expansión restringida del mercado, democratización para minorías y renovación en las ideas pero con eficacia mínima en los procesos sociales, pese al pujante quehacer del movimiento obrero (Soto, 2004). Amanda planteó que nuestra República productora de hierro, cobre, nitrato y carbón, ostentaba los elementos indispensables en la economía bélica, los cuales aceleraron el ritmo de las exportaciones y por ende de su producción, lo cual permitió que Chile tuviera una pasajera elevación de su economía durante el periodo bélico, aunque una sensible disminución en el tiempo de posguerra (Labarca, 1953).

Específicamente, en la década entre 1930 y 1940, se desarrollaron diversos movimientos en pro de los derechos de las mujeres en materia civil y legislativa que consagraron los numerosos esfuerzos gestados desde finales del siglo XIX (Ríos Tobar, 2009). En este contexto, Amanda tuvo un destacado rol en la discusión y reivindicación de los derechos de las mujeres, a través de su contribución en el Consejo Nacional de Mujeres, grupo destinado al debate sobre los derechos femeninos (Reyes, Vargas, \& Meza, 2002), del cual posteriormente sería elegida presidenta, lo que sumado a su participación en el Partido Radical (Muñoz, 1998), le permitió tomar acción política en la lucha por los derechos de las mujeres (Galarce \& Cortés, 1986; Labarca, 1946), uno de los temas sociopolíticos centrales en la vida de la educadora.

Esta temática estuvo fuertemente influida por los acelerados cambios del mundo contemporáneo en cuanto a las libertades individuales y la igualdad de derechos (Salas, 1996). Amanda estaba imbuida de un inmenso idealismo, lo cual daba cuenta de la fe que tenía en la obra futura de la mujer chilena y su despertar a una conciencia nacional que le permitiera generar grandeza en su país (Labarca, 1914). El resultado más relevante de este proceso fue la elaboración del primer proyecto de ley que reivindica derechos civiles a las mujeres en Chile y posteriormente las leyes que les permitirán votar en elecciones municipales en 1934 y en las elecciones parlamentarias y presidenciales en 1949.

\section{El tránsito de lo alemán al pragmatismo norteamericano}

Durante el siglo XIX, la cultura francesa pierde fuerza en Chile, y al mismo tiempo Alemania se presenta como nación de "cultura e innovadora" (Alfonso \& Pacheco, 2011). El predominio de la pedagogía alemana en Chile tuvo su apogeo en la última década del siglo XIX. Desde 1885, esta influencia inspiró las escuelas normales y a través de estas a la primera enseñanza. Los profesores germanos que dirigían el Instituto Pedagógico de la Universidad de Chile y algunos maestros chilenos tan influyentes como Barros Arana, aunaron sus esfuerzos para implementar en los liceos, junto a los principios de la didáctica propuesta por Herbart, los planos y métodos concéntricos. Pese a la campaña doctrinaria, sustentada con más talento que ciencia por Eduardo de La Barra, la influencia alemana fue entonces indiscutible e indiscutida (Labarca, 1927a). Con el paso de los años, Amanda se fue 
transformando en la embajadora chilena en Estados Unidos, y muestra de ello fue su presentación en el marco del Congreso Panamericano de Pedagogía, realizado en Washington en el mes de Mayo de 1940, donde dio a conocer el rol del Instituto Pedagógico, enalteciendo su función educadora en la formación del carácter y la implantación de los ideales superiores en la más alta calidad (Labarca, 1940). Con esto queda en evidencia que la labor del Pedagógico iniciada a fines del siglo XIX en Chile, extendía sus fronteras y no solo iba a ser conocido en América Latina y Alemania, sino también en Norteamérica.

Antes que descubrimientos valiosos, lo que consiguió la psicología en la primera mitad del siglo XIX fue desprenderse del cúmulo de errores que sobre ella habían amontonados siglos de estudio. Escasamente, la psicología general y la especial del niño lograban reunir observaciones suficientes para construir hipótesis más valederas. Por su parte, a falta de base científica, la pedagogía fue por mucho tiempo, una creencia, una fe (Labarca, 1927a), y en este contexto la psicología comenzó a prestar el auxilio que la pedagogía necesitaba. En las postrimerías del siglo XIX, en uno de los primeros textos de psicología educacional que se conoce, Sully (1899) argüía que, de las ciencias que proporcionaban principios a la educación, la más importante era la psicología y lo que más interesaba al maestro se relacionaba con el desarrollo de la mente del educando.

Volviendo a Chile, se entreveía que los problemas sociales, entre los cuales el de la educación ocupaba un lugar destacado, no se podrían resolver con la mera implementación de programas foráneos. En rigor, se estaba asistiendo a una emancipación respecto de la influencia europea. Por otra parte, se estaba produciendo un desplazamiento del pensamiento alemán y francés hacia el norteamericano. En ese contexto, algunos profesores fueron a capacitarse a Estados Unidos y también se importaron varios recursos humanos, como algunas directoras de escuelas normales (Labarca, 1927a). Según Pacheco (2012), el nuevo siglo XX vino acompañado de una renovación en el campo pedagógico, donde se constataría la influencia de autores norteamericanos como John Dewey.
Durante aquellos primeros años del nuevo siglo, William James se convertía en uno de los pilares de la psicología y la educación a nivel mundial. Desde luego, su influencia y su obra Pragmatismo de 1907 (James, 1952) no pasaron inadvertidos en América Latina. En gran medida, la psicología pragmatista de James y el movimiento positivista fueron el soporte teórico y la antesala del conductismo watsoniano (Salas, 2013), el cual tuvo un impacto decisivo no solo en Estados Unidos, sino también en el mundo entero. En todo este contexto, Amanda leyó con prisa y aceptación a James y Dewey, junto a otros pensadores estadounidenses. Varias de sus ideas nutrieron el trabajo de Amanda y como toda corriente de pensamiento, en algún momento la atracción norteamericana hizo su eclosión a favor de otras corrientes y en razón de la propia complejidad del terreno pedagógico en Chile.

\section{La influencia norteamericana: James y Dewey}

Salas (1995) señaló que las aspiraciones de Amanda, Darío Salas y otros educadores en materia de pedagogía y educación, estaban fuertemente influidas por los principios educacionales de Estados Unidos. Si estos educadores comprendían las diferencias entre ambas sociedades, buscaron la forma de adaptar a nuestra realidad las ideas que consideraban adecuadas para el país. En 1910, Amanda pudo cumplir sus deseos de realizar estudios en Estados Unidos e ingresó al afamado Teachers College de la Universidad de Columbia en Nueva York, donde tuvo la oportunidad de seguir cursos con grandes personeros de la educación de la época, entre los que figuraban Dewey, Kirkpatrick, Thorndike y otros (Salas, 1996). Durante dos años, tomó cursos en la Universidad de Columbia en Estados Unidos, tiempo cuando el citado pragmatismo adquiría cada vez más una amplia repercusión en el mundo de la psicología y la pedagogía (Portales, 1990; Portugal, 1993) que, sumado al intuicionismo de Bergson, trató de orientar la pedagogía de principios de la centuria pasada (Labarca, 1927a).

La influencia pedagógica de James, debía atribuirse más a su calidad de psicólogo que de filósofo. 
Su gran obra Principios de psicología y posteriormente el Compendio, que él mismo redactó para la lectura y uso de un público no especializado, popularizaron en vasta escala los descubrimientos de la psicología e introdujeron a los maestros en temas psicológicos. Todo esto aconteció sobre las investigaciones experimentales de Wundt en Alemania, de Stanley Hall sobre la adolescencia en Estados Unidos y de Binet sobre los niños escolares en Francia. Estos desarrollos precedieron a los trabajos contemporáneos de Claparéde, Thorndike y tantos otros que concurrieron a cimentar sólidamente la psicología (Labarca, 1927a).

Sin embargo, James, fue inspirador no solo de Dewey, sino de una extensísima falange de maestros de las nuevas escuelas en Estados Unidos (Labarca, 1927a), lo cual se denota en Los ideales de la vida (James, 1904), donde se compilan conferencias dictadas a los maestros de Cambridge y otros discursos a los jóvenes realizados en la Escuela Normal de Gimnástica de Boston. Amanda planteó que en James se advertía la tendencia a subrayar en la psicología del niño la importancia de los fenómenos emotivos en el ejercicio de los hábitos y la construcción del carácter, disminuyendo el énfasis colocado con anterioridad sobre los procesos puramente intelectuales (Labarca, 1927a). A diferencia de James, la influencia de Dewey fue mucho más vasta como filósofo y doctrinador de pedagogos que como psicólogo. De acuerdo con sus teorías pragmáticas e instrumentalistas "la educación es una función social, que asegura la dirección y el desarrollo de los seres inmaduros mediante su participación en la vida del grupo al que pertenecen" (Dewey, 1971, p. 92). Para Dewey, la esencia de la teoría educativa se centra en la interacción y cooperación entre el niño como ser inmaduro, no desarrollado y la experiencia madura del adulto que transmite ciertos fines sociales, ideas y valores (Dewey, 1959).

Amanda dedicó un amplio apartado a la obra de Dewey en Nuevas Orientaciones de la enseñan$z a$, trazando su diferencia con la gran mayoría de los pedagogos. Bajo la directriz del pensamiento de Dewey, señala que los conocimientos no pueden transmitirse, sino que se adquieren por medio de la experiencia, a través de una relación vital entre el individuo y el "aspecto" que se desea conocer (Labarca, 1927a). La admiración de Amanda sobre Dewey se revela cuando plantea que su doctrina implicaba revolucionar los métodos, la disciplina, los programas e incluso el local y el mobiliario de los colegios que se habían construido para la transmisión oral de los conocimientos (Labarca, 1927a).

\section{Lo psicológico en "Lecciones de Filosofía"}

No es intención de este trabajo referirse en detalle a la totalidad de los conocimientos psicológicos planteados por Amanda, sino esbozar las nociones centrales para comprender en qué tenor se encontraba su mirada sobre la disciplina psicológica. En el tomo II de su libro Lecciones de Filosofía (Labarca, 1927b) dedicó amplios apartados a estructurar conceptos relevantes para la psicología. Dicho trabajo tuvo por objeto facilitar a los alumnos de quinto y sexto año de humanidades el estudio de la filosofía. En el citado trabajo, señaló que los apuntes dictados por los profesores tenían grandes inconvenientes y aunque su obra fuera imperfecta reemplazaría con ventaja a las preexistentes. Agregó que la razón de ser del libro no se relacionaba con poseer méritos de originalidad, ya que su intención era prestar una ayuda en el aprendizaje de la asignatura, hasta que se redactaran los textos definitivos de los cuales se carecía. El libro se encuentra estructurado en tres partes: la primera dedicando un amplio apartado a la psicología, la segunda a la lógica y la tercera a la historia de la filosofía. En lo concerniente a la psicología, dividió los hechos psicológicos en tres grandes grupos: intelectuales o representativos, sensitivos o afectivos y volitivos o activos. Declaró que los primeros tienen relación con la inteligencia, los segundos con el sentimiento y los terceros con la voluntad (Labarca, 1927b). Para realizar este trabajo, la aludida conocía trabajos clásicos como El tratado de las pasiones (Descartes, 1649/2005). En relación con esta obra, apuntó que Descartes no se limitó -como otros pensadores antiguos- a hacer consideraciones más o menos generales sobre 
nuestros diversos sentimientos, sino que trató de explicarlos, relacionándolos con las modificaciones orgánicas que los acompañan y con las cuales se conectan. En relación con la vida intelectual y afectiva, esbozó algunas diferenciaciones relevantes, dando cuenta de que los fenómenos de orden intelectual serían más precisos y distintos al diferir una idea de otra, como difieren nuestros juicios y raciocinios; lo que contrastó con la vida afectiva donde las situaciones que en algún momento nos complacen en cualquier otro momento pueden disgustarnos, transformándose de esta forma lo agradable en desagradable. Para ello se refirió también al concepto de sensibilidad, planteando que es por esta que nos interesamos sobre la vida que vivimos y en la de nuestros semejantes, pudiendo sentir pena o placer como aspirar a una vida más intensa (Labarca, 1927b). En la construcción de sus ideas, se observan referencias relacionadas con tratados de lógica (Goblot, 1921; Rey, 1900; Stuart Mill, 1911), filosofía (Janet, 1904), psicología (Dewey, 1916; James, 1916; Rey, 1926) y pedagogía (Compayré, 1887), aunque todos esos textos derivaran en temáticas psicológicas.

En el prólogo del Tratado elemental de Filosofía, el psicólogo francés Pierre Janet planteó que "la psicología, la lógica, la moral y la estética forman una sola ciencia que es la ciencia del hombre y no debe cortarse por la mitad" (Janet, 1904, p. vii); incluso señaló que la psicología era una parte de la filosofía que trataba del estudio el espíritu humano y de sus facultades, para lo que dedicó más de 300 planas al tratamiento de temáticas tales como la percepción, memoria, pensamiento, imaginación, emociones, voluntad, entre otros procesos y fenómenos psicológicos. Casi al mismo tiempo, otros como Wundt y Titchener intentaban hacer de la psicología una ciencia independiente, logrando gestar una psicología experimental.

Las lecturas, experiencias y formación académica de Amanda tanto en Chile como en Estados Unidos, unida a su espíritu crítico y con pretensión de conocimiento permitieron que estuviera al tanto de algunas de las más importantes referencias psicológicas de fines del siglo XIX e inicios del XX.

\section{Conclusiones}

La obra de Amanda Labarca ha permanecido recóndita en los anaqueles de la historia de la cultura chilena. Si bien un liceo en Santiago lleva su nombre y aun cuando se han realizado diversos homenajes a su persona, sigue siendo necesario conocer su trabajo académico. Es interesante retroceder en el tiempo para prestar atención a diversos detalles de su obra en temáticas relacionadas con el rol público de la mujer, la educación y la psicología, entre otros temas que abordó.

Si bien las contribuciones que realizó Amanda a la psicología no fueron originales, no obstante permitieron el acercamiento de esta ciencia como disciplina auxiliar a la formación de pedagogos. Sus referencias psicológicas fueron principalmente norteamericanas por sus constantes visitas a Estados Unidos, y como se mencionara, los autores que más influyeron en su formación fueron William James y John Dewey, de quienes recibió su pensamiento.

Hay que destacar que con su trabajo, imprimió un cambio a la influencia europea (Salas, 2012) que ostentaba la psicología chilena desde fines del siglo XIX, trasladando el centro de atención hacia Estados Unidos, como también ocurrió en otras naciones del mundo.

En el epílogo, es relevante precisar que otros aspectos de la vida de Amanda, podrán ser profundizados en trabajos venideros, por ejemplo, lo concerniente a esclarecer su vida privada, pública y social (González Vera, 1967), todo lo cual permitirá comprender y valorar de mejor forma la obra nuestra de gran educadora.

\section{Referencias}

Alfonso, A., \& Pacheco, S. (2011). Elisabeth Isabel Bongard. Migrante y protagonista de la reforma educacional en Chile. La Serena: ULS.

Ardila, R. (1973). La psicología en Colombia: desarrollo histórico. México: Trillas.

Ardila, R. (1988). Mercedes Rodrigo (1891-1982). Revista Latinoamericana de Psicología, 20(3), 429-434.

Ascolani, A. (1988). Psicología en Rosario. Una crónica de recuerdos y olvidos. Rosario: Ross. 
Barr-Melej, P. (2001). Reforming Chile: Cultural, politics, nationalism and the rise of the middle class. Chapel Hill, NC: University of North Carolina Press

Bohan, J. (1993a). Women at center stage: A course about the women of psychology. Teaching of Psychology, 20(2), 74-79.

Bohan, J. (1993b). Regarding gender: Essentialism, constructionism, and feminist psychology. Psychology of Women Quarterly, 17(1), 5-21.

Carpintero, H. (2012). Fernanda Monasterio, una voz española en la Sociedad Interamericana de Psicología (SIP). Revista Interamericana de Psicología, 46(1), 43-50.

Compayré, G. (1887). Lectures on pedagogy, theoretical and practical. Boston: D.C. Heath \& Co.

Dager, J. (2003). Poner en claro los hechos al escribir la historia: la metodología de la investigación del pasado de Andrés Bello. Revista Electrónica de Historia, 3, 2-28.

De la Fuente, D. (30 de junio de 1979). Sobre Amanda Labarca. La tribuna de Los Ángeles, p. 3.

De la Fuente, D. (7 de septiembre de 2000). Amanda Labarca. Diario El Angolino, p. 3.

Descartes, R. (2005). Tratado de las pasiones del alma. Madrid: Editorial Edaf. (Trabajo original publicado en 1649)

Dewey, J. (1916). Psicología del pensamiento. Boston: Sin editorial.

Dewey, J. (1959). On education. New York: Bureau of Publications.

Dewey, J. (1971). Democracia y educación. Buenos Aires: Editorial Losada.

Fernández, B. (2007). El cuerpo-máscara de Amanda Labarca. Poderes que muestran y ocultan el feminismo chileno. Primera mitad del siglo XX. Nuestra Historia, 2, 123-140.

Galarce, G., \& Cortes, W. (11 de diciembre de 1986). Amanda Labarca, impulsora de los derechos de la mujer. La Estrella de Arica, p. 2.

Gallegos, M., \& Berra, M. (2012). La psicología en Argentina: 25 años de la Facultad de Psicología (UNR). Cuadernos Sociales, 12, 123-152.

Gallegos, M. (2005). Cincuenta años de historia de la psicología como institución universitaria en Argentina. Revista Latinoamericana de Psicología, 37(3), 641-652.
García, S. (2003). Psicología y feminismo: una aproximación desde la psicología social de la ciencia y las epistemologías (Memoria para optar al grado de doctor). Universidad Complutense de Madrid, España.

García, S. (2005). Mary Whiton Calkins: la psicología como ciencia del self. Athenea Digital, 8, 81-28.

García, S. (2010). El olvido de las mujeres pioneras en la historia de la psicología. Revista de Historia de la Psicología, 31(4), 9-22.

Goblot, E. (1921). Traité de logique. Paris: Libr. de la Vda. de Ch. Bouret.

González Vera, J. (1967). Algunos. Santiago: Editorial Nascimento.

Guil, A., \& Vera, S. (2010). Amanda Labarca, pionera de la educación y la política de las mujeres en Chile. Revista Historia de la Educación Colombiana, 13, 143-159.

Jacó-Vilela, A., Messias, M., Oliveira, F., Espírito Santo, A., Carneiro, F., \& Valente, N. (2007). Uma gestação silenciosa: a presença feminina nas instituições de psicologia no Rio de Janeiro na primeira metade do século XX. En M. Ribeiro, J. Bernardes \& C. Lang (Orgs.), A produção na diversidade: compromissos éticos e políticos em psicologia (Vol. 1, pp. 231-266). São Paulo: Casa do Psicólogo.

Jacó-Vilela, A., Santos, V., \& Barbosa, C. (2008). Mulheres do laboratório de biologia infantil. Boletim do CDPHA, 20, 89-92.

James, W. (1904). Los ideales de la vida. Barcelona: Editores Henrich y Cía.

James, W. (1916). Compendio de psicología. Madrid: Daniel Jorro Editor.

James, W. (1952). Pragmatismo. Buenos Aires: Emecé Editores.

Janet, P. (1904). Tratado elemental de Filosofía. Para uso de los establecimientos de enseñanza. París: Bouret.

Labarca, A. (1914). Actividades femeninas en los Estados Unidos. Santiago: Imprenta Universitaria.

Labarca, A. (1919). La enseñanza secundaria en los Estados Unidos. Santiago: Imprenta Universo.

Labarca, A. (1927a). Nuevas orientaciones de la enseñanza. Santiago: Imprenta Universitaria.

Labarca, A. (1927b). Lecciones de Filosofía. Santiago: Sociedad Imprenta y Litografía Universo.

Labarca, A. (1936). Mejoramiento de la vida campesina. Santiago: Unión Republicana. 
Labarca, A. (1938). Evolución de la segunda enseñanza. Santiago: Nascimento.

Labarca, A. (1939). Historia de la enseñanza en Chile. Santiago: Publicaciones de la Universidad de Chile.

Labarca, A. (1940). Educación del adolescente. [Trabajo presentado en el Congreso Científico Panamericano en Washington]. Revista Atenea, Tomo LXII.

Labarca, A. (1944). Bases para una política educacional. Buenos Aires: Losada.

Labarca, A. (2 de marzo de 1946). Cable a Gabriela Mistral. Comercial Cables, Cablegram. The Comercial Cable Company, p. 7.

Labarca, A. (1947). Feminismo contemporáneo. Santiago: Zig Zag.

Labarca, A. (1953). Realidades y problemas de nuestra enseñanza. Santiago: Universitaria.

Magaña, I., Wolff, X., \& Winkler, M. I. (1998). Historia de la psicología y género: percepción de los estudiantes de psicología. Revista Chilena de Psicología, 19(2), 32-42.

Miranda, M., \& Navarro, M. (1995). La historia de la psicología en Chile (Tesis conducente al grado de licenciado en psicología). Pontificia Universidad Católica de Chile, Santiago, Chile.

Moradi, B., \& Townsend, D. T. (2006). Raising students' awareness of women in psychology. Teaching of Psychology, 33(2), 113-117.

Munizaga, R. (1991). Amanda Labarca Hubertson. Societas, 1(1), 307-310.

Muñoz, M. (1 de marzo de 1998). La educadora Amanda Labarca. El Magallanes, p. 3.

Núñez, I. (2002). La historia de la enseñanza en Chile. Páginas Didácticas, 9, 91-93.

Ostrovsky, A. (2010). Las historias críticas de la psicología desde la perspectiva de género: pensando sus influencias y posibilidades. Estudos e Pesquisas en Psicologia, 10(3), 911-929.

Pacheco, S. (2012). Sembrando abecedarios. Historia, pedagogía y asistencia en la educación primaria rural, región de Coquimbo. Coquimbo: SALC.

Palma, L. (9 de enero de 1976). Amanda Labarca Hubertson. La Tercera de la Hora, p. 3.

Pinto, P. (1990). El paradigma masculino-femenino en el discurso narrativo de Amanda Labarca. Acta Literaria, 15, 133-146.
Portales, P. (28 de octubre de 1990). Amanda Labarca Hubertson. Artesana de la Tolerancia. Diario La Nación, p. 6.

Portugal, A. (19 de abril de 1993). Una Amanda Labarca transgresora. Marea Alta, p. 12.

Quintana, J., \& Feldman, R. (2007). Apunte biográfico sobre F. Monasterio, Fundadora de la sección de psicología de la Facultad de Humanidades de la Universidad Nacional de La Plata (Argentina). Boletín de la Sociedad Española de Historia de la Psicología, 39, 5-15.

Rey, A. (1900). Lógica. Madrid: Espasa-Calpe.

Rey, A. (1926). Psicología. Madrid: Espasa-Calpe.

Reyes, M., Vargas, H., \& Meza, C. (2002). Recordando a Amanda: Bautizada Pinto, renombrada Labarca. Psykhe, 11(1), 117-128.

Ríos Tobar, M. (2009). When are women's movements feminist? Framing and movement cycles in Chile since the 1930s (Disertación doctoral). University of Wisconsin, Madison, Estados Unidos.

Salas, E. (1995). La señora Amanda, mi padre y sus amigos. Revista Occidente, 353, 25-28.

Salas, E. (1996). Amanda Labarca. Dos dimensiones de la personalidad de una visionaria mujer chilena. Santiago: Ediciones Mar del Plata.

Salas, G. (2012). La influencia europea en los inicios de la historia de la psicología en Chile. Revista Interamericana de Psicología, 46(1), 99-110.

Salas, G. (2013). Juan Serapio Lois (1844-1913) pionero de la psicología científica en Chile. Psykhe, 22(1), 111-123.

Salas, G., \& Lizama, E. (2013). Historia de la psicología en Chile. 1889-1981 (2.a ed.). La Serena: Editorial Universidad de La Serena.

Scott, M., Brown, F., Marshall, K., Judd, E., Braboy, L., \& Jhaveri-Metha, S. (2012). Women psychologist: Multiple paths, similar yet distinct identities. Journal of Humanistic Psychology, 52(3), 279-303.

Soto, M. (2004). Políticas educacionales en Chile durante el siglo XX. Revista Mad. Departamento de Antropología, 10, 1-13.

Stuart Mill, J. (1911). Resumen sintético del sistema de lógica. París: Libr. de la Vda. de Ch. Bouret.

Sully, J. (1899). Psicología pedagógica. New York: D. Appleton y Cia. 
Vargas, P. (2006). Tres grandes mujeres en la historia de Chile. Revista Arauco, 2, 28-29.

Winkler, M. I. (2004). Pioneras sin monumentos: mujeres en la historia de la psicología en Norteamérica, Argentina y Chile durante las tres primeras décadas del desarrollo de la disciplina (Tesis para optar al grado de Doctora en Estudios Americanos). Universidad de Santiago de Chile, Chile.

Winkler, M. I. (2007). Pioneras sin monumentos. Mujeres en psicología. Santiago: LOM.

Winkler, M. I. (2012). Historia de mujeres. Presencias y ausencias en la Sociedad Interamericana de Psicología. Revista Interamericana de Psicología, 46(1), 67-78.
Winkler, M. I., Magaña, I., \& Wolff, X. (2001). Autorías y paradojas: mujeres en la historia de la psicología. Revista Latinoamericana de Psicología, 33(1), 23-37.

Winkler, M. I., Magaña, I., \& Wolff, X. (2004). Otra historia de la psicología: una triada de mujeres. Revista Interamericana de Psicología, 38(1), 5-14.

Winkler, M. I., Pasmanik, D., Wolff, X., \& Reyes, M. (2007). Ética y género en la obra de pioneras y pioneros de la psicología en Estados Unidos de Norteamérica y Chile. Revista Latinoamericana de Psicología, 39(3), 523-535.

Wolff, X., Winkler, M. I., \& Alvear, K. (2010). Subversiones de género: pioneras de la psicología chilena y estadounidense. Psychologia Latina, 1(2), 129-138. 